Pacific Journal of Mathematic 


\section{CLASSES DE CHERN ET THEOREME DE GAUSS-BONNET}

\section{NGô VAN QUÊ}

La theorie des classes caractéristiques peut etrê développée de manière axiomatique dans la catégorie des espaces topologiques "admissibles", i.e., localement compacts, dénombrables á l'infini et de dimension cohomologique finie [Hirzebruch, Topological Methods in Algebraic Geometry, Springer Verlag, N.Y.]. Nous allons nous restreindre à la catégorie des variétés paracompactes, différentiables de classe $\mathscr{C}^{\infty}$ et introduire les classes caractéristiques telles qu'clles étaient construites originalement par S. Chern [voir par exemple, S. Chern, Differential geometry of fiber bundles, Proc. Int. Congress, 1950]. P. A. Griffiths a démontré le théoremè, dû à Chern, dit de Gauss-Bonnet pour le cas des fibrés vectoriels de rang 1 [On a theorem of Chern, Illinois J. Math. 6 (1962)]. Dans le même ordre d'idées nous voulons donner dans ce travail une démonstration simple, par la théorie des connexions, du théorème de Gauss-Bonnet dans le cas des fibrés vectoriels de rang quelconque.

1. Connexion dans un fibre hermitien.

1. Soit $M$ une variété (différentiable). Le fibré tangent de $M$ sera noté par $T$. Le fibré $T_{C}^{*}=T^{*} \bigotimes_{R} C$ désignera le fibré des 1-formes complexes sur $M$.

DÉfinition 1.1. Etant donné un fibré vectoriel complexe $E$ sur $M$, une connexion dans $E$ est la donnée d'un opérateur différentiel

$$
\nabla: E \longrightarrow T_{C}^{*} \otimes E
$$

(produit tensoriel de Whitney sur le corps des nombres complexes), tel que pour toute fonction $f$ à valeurs complexes sur $M$

$$
\nabla(f s)=d f \otimes s+f \nabla(s) ;
$$

dans le cas où $E$ est muni d'une structure hermitienne, la connexion sera dite hermitienne si

$$
d\left\langle s, s^{\prime}\right\rangle=\left\langle\nabla(s), s^{\prime}\right\rangle+\left\langle s, \nabla\left(s^{\prime}\right)\right\rangle .
$$

ExEmPle. Considérons le fibré hermitien trivial

$$
E=M \times C^{n} .
$$

Toute section $s$ de $E$ sera un $n$-tuple $\left(f^{1}, \cdots, f^{n}\right)$ de fonctions sur $M$ à valeurs complexes, et on a $T_{c}^{*} \otimes E=T_{c}^{*} \times \cdots \times T_{C_{-}}^{*}$, produit $n$-tuple 
direct de Whitney. Alors l'operateur $\nabla$ défini par

$$
\nabla\left(f^{1}, \cdots, f^{p}\right)=\left(d f^{1}, \cdots, d f^{p}\right)
$$

est évidemment une connexion hermitienne dans $E$.

Par cet exemple, et de l'existence des partitions de 1' unité sur $M$, on démontre immédiatement (voir tout cours de géométrie différentielle) l'existence des connexions hermitiennes sur tout fibré vectoriel complexe $E$ sur $M$.

Sur un ouvert $U$ de trivialisation de $E$, l'ensemble des $n$ sections $s^{1}, \cdots, s^{n}$ de $E$ est une base locale de $E$ si toute autre section $s$ de $E$ sur $U$ est de la forme

$$
s=f_{i} s^{i} \text { avec } f_{i} \in \mathscr{E}^{\infty}(U, C)
$$

(nous prenons ici comme dans toute la suite la convention d'Einstein de sommation). Alors pour une connexion $\nabla$ dans $E$, on a

$$
\nabla\left(s^{i}\right)=\omega_{j}^{i} \otimes s^{j}
$$

$\omega_{j}^{i}$ étant des 1-formes complexes sur $U$. La matrice $n \times n\left(\omega_{j}^{i}\right)=\omega$ dont les entrées sont des 1-formes complexes, sera dite la matrice représentative de $\nabla$ relativement à la base locale $\left(s^{1}, \cdots, s^{n}\right)$. Cette matrice définit inversement l'opérateur $\nabla$ restrient à $U$ par

$$
\nabla(s)=\nabla\left(f_{i} s^{i}\right)=d f_{i} \otimes s^{i}+f_{i} \omega_{j}^{i} \otimes s^{j} .
$$

Rappelons enfin que si $E$ est muni d'une connexion $\nabla$, on peut définir sur l'espace fibré vectoriel dual $E^{*}$ une connexion canonique notée encore par $\nabla$ telle que si $s$ et $s^{\prime}$ sont respectivement des sections de $E$ et de $E^{*}$

$$
d\left(s, s^{\prime}\right)=\left(\nabla(s), s^{\prime}\right)+\left(s, \nabla\left(s^{\prime}\right)\right) .
$$

Et soit $\left(E_{i}\right)$ avec $i=1, \cdots, p$, un ensemble de $p$ fibrés vectoriels complexes sur $M$, munis respectivement des connexions $\nabla_{i}$. On peut définir sur $E_{1} \otimes \cdots \otimes E_{p}$ une connexion canonique $\nabla$ telle que

$$
\begin{aligned}
\nabla: E_{1} \otimes \cdots \otimes E_{p} & \longrightarrow T_{C}^{*} \otimes\left(E_{1} \otimes \cdots \otimes E_{p}\right) \\
s^{1} \otimes \cdots \otimes s^{p} & \longrightarrow s^{1} \otimes \cdots \otimes s^{i-1} \otimes \nabla_{i}\left(s^{i}\right) \otimes s^{i+1} \otimes \cdots \otimes s^{p}
\end{aligned}
$$

en remarquant l'existence d'un isomorphisme cononique

$$
\begin{aligned}
T_{C}^{*} \otimes\left(E_{1} \otimes \cdots \otimes E_{p}\right) & \simeq E_{1} \otimes \cdots \otimes E_{i-1} \otimes\left(T_{C}^{*} \otimes E_{i}\right) \\
& \otimes E_{i+1} \otimes \cdots \otimes E_{p} .
\end{aligned}
$$

2. Tenseur de courbure. Etant donnée une connexion $\nabla$ dans le fibré vectoriel $E$, on peut définir de manière générale pour tout 
entier $p$

$$
\nabla: \frac{\left(\Lambda^{p} T_{C}^{*}\right) \otimes E}{\theta \otimes s} \longrightarrow \frac{\left(\Lambda^{p+1} T_{C}^{*}\right) \otimes E}{d \theta \otimes s+(-1)^{p} \theta \Lambda \nabla(s)} .
$$

Il est immédiat par un simple calcul que

$$
\nabla^{2}=\nabla \circ \nabla: \frac{\left(\Lambda^{p} T_{C}^{*}\right) \otimes E}{\theta \otimes s} \longrightarrow \frac{\left(\Lambda^{p+2} T_{C}^{*}\right) \otimes E}{\theta \Lambda \nabla^{2}(s)}
$$

L'opérateur $\nabla^{2}$ est donc un morphisme de fibrés vectoriels; en particulier:

$$
\nabla^{2}: E \longrightarrow\left(\Lambda^{2} T_{C}^{*}\right) \otimes E
$$

peut-être considéré comme une section globale $\Omega$ sur $M$ du fibré $\left(\Lambda^{2} T_{C}^{*}\right) \otimes$ End $(E) . \quad \Omega$ est couramment nommé tenseur de courbure de la connexion $\nabla$.

Si $\nabla$ est une connexion hermitienne de $E$, on a

$$
d\left\langle s, s^{\prime}\right\rangle=\left\langle\nabla(s), s^{\prime}\right\rangle+\left\langle s, \nabla\left(s^{\prime}\right)\right\rangle
$$

donc

$$
d^{2}\left\langle s, s^{\prime}\right\rangle=d\left\langle\nabla(s), s^{\prime}\right\rangle+d\left\langle s, \nabla\left(s^{\prime}\right)\right\rangle
$$

ou

$$
\begin{aligned}
& 0=\left\langle\nabla^{2}(s), s^{\prime}\right\rangle-\left\langle\nabla(s), \nabla\left(s^{\prime}\right)\right\rangle+\left\langle\nabla(s), \nabla\left(s^{\prime}\right)\right\rangle+\left\langle s, \nabla^{2}\left(s^{\prime}\right)\right\rangle \\
& 0=\left\langle\nabla^{2}(s), s^{\prime}\right\rangle+\left\langle s, \nabla^{2}\left(s^{\prime}\right)\right\rangle .
\end{aligned}
$$

Autrement dit, avec la notation habituelle.

Lemma 1. Si $\nabla$ est une connexion hermitienne, on a

$$
\Omega+{ }^{t} \bar{\Omega}=0 .
$$

D'après la fin de la section précédente, nous avons défini à partir de la connexion $\nabla$, une connexion canonique notée encore par $\nabla$, dans End $(E)=E \otimes E^{*}$. Précisément si $a$ est une section de $E \otimes E^{*}$ et $s$, une section de $E$,

$$
[\nabla(a)](s)=\nabla[a(s)]-a[\nabla(s)]
$$

avec une interprétation immédiate de notations. Ceci dit.,

Lemma 2. (Identité de Bianchi.)

$$
\nabla(\Omega)=0 \text {. }
$$

En effet, d'après la définition même de $\nabla$ dans End $(E)$, on a 


$$
\nabla(\Omega)=\nabla \circ \nabla^{2}-\nabla^{2} \circ \nabla=0
$$

comme opérateurs sur $\bigoplus_{p} \Lambda^{p} T_{c}^{*} \otimes E$.

Remarque. Donnée une base locale $\left(s^{1}, \cdots, s^{n}\right)$ de $E$, on a

$$
\Omega\left(s^{i}\right)=\nabla^{2}\left(s^{i}\right)=\Omega_{j}^{i} \otimes s^{j}
$$

avec $\Omega_{j}^{i}$, des 2-formes complexes sur l'ouvert $U$ de trivialisation correspondant de $E$. La matrice carrée $\mathrm{n} \times n\left(\Omega_{j}^{i}\right)=K$. Alors si $\omega=\left(\omega_{j}^{i}\right)$ est la matrice représentative de $\nabla$, l'identité de Bianchi se traduit par l'identité

$$
0=d K+[\omega, K]
$$

i.e.,

$$
0=d \Omega_{j}^{i}+\omega_{k}^{i} \wedge \Omega_{j}^{k}-\Omega_{k}^{i} \wedge \omega_{j}^{k}, \forall i \text { et } j
$$

3. Famille de connexions. Une famille de connexions $\nabla_{t}$ sur $E$, dépendant du paramètre réel $t \in[0,1]=I$, sera toujours entendue telle que pour toute section $s$ de $E$

$$
\sigma_{t}=\nabla_{t}(s)
$$

est une famille différentiable $\mathscr{E}^{\infty}$ de sections de $T_{c}^{*} \otimes E$, i.e., l'application

$$
\begin{gathered}
\sigma: M \times I \longrightarrow T_{C}^{*} \otimes E \\
(x, t) \longrightarrow \sigma_{t}(x)
\end{gathered}
$$

est différentiable $\mathscr{C}^{\infty}$ (morphisme de notre catégorie).

Etant donnée une telle famille de connexions $\nabla_{t}$, considérons l'opérateur

$$
\begin{aligned}
\dot{\nabla}_{t}: E & \longrightarrow T_{C}^{*} E \\
& \longrightarrow \frac{\partial}{\partial_{t}}\left[\nabla_{t}(s)\right]=\dot{\nabla}_{t}(s) .
\end{aligned}
$$

On a pour toute fonction $f$ à valeur complexe de $M$

$$
\dot{\nabla}_{t}(f s)=\frac{\partial}{\partial_{t}}\left[d f \otimes s+f \nabla_{t}(s)\right]=f \dot{\nabla}_{t}(s) .
$$

Donc $\dot{\nabla}_{t}$ peut être considéré comme une section de $T_{C}^{*} \otimes$ End $(E)$. Comme précédemment, à la famille $\nabla_{t}$, il correspond une famille de tenseurs de courbure $\Omega_{t}$ (i.e., une famille différentiable de sections de $\Lambda^{2} T_{c}^{*} \otimes$ End $\left.(E)\right)$ et une famille de connexions notée encore par $\nabla_{t}$ dans End $(E)$. Ceci dit, 
LEMMA 3.

$$
\frac{\partial}{\partial_{t}}\left(\Omega_{t}\right)=\nabla_{t}\left(\dot{\nabla}_{t}\right)
$$

En effet, comme opérateur de $E$ dans $\Lambda^{2} T_{C}^{*} \otimes E$,

$$
\frac{\partial}{\partial_{t}}\left(\Omega_{t}\right)=\frac{\partial}{\partial_{t}}\left(\nabla_{t}^{2}\right)=\nabla_{t} \circ \dot{\nabla}_{t}+\dot{\nabla}_{t} \circ \nabla_{t}=\nabla_{t}\left(\dot{\nabla}_{t}\right)
$$

la dernière égalité provenant de la définition de $\nabla_{t}$ dans End $(E)$.

\section{Classes de Chern.}

1. Nous pouvons évidemment considérer

$$
\left(\Lambda^{p \alpha i k} T_{C}^{*}\right) \otimes E=\bigoplus_{p}\left(\Lambda^{2 p} T_{C}^{*}\right) \otimes E
$$

comme fibré de modules libres sur le fibré en algèbre commutative

$$
\Lambda^{p \alpha i k} T_{C}^{*}=\bigoplus_{p} \Lambda^{2 p} T_{C}^{*}
$$

Alors $\Omega=\nabla^{2}$ est un endomorphisme de ce fibré de modules. Donc nous pouvons considérer comme d'habitude $\operatorname{det}(I+(i / 2 \pi) \Omega)$, qui sera donc une section globale sur $M$ du fibré $\Lambda^{p \alpha i R} T_{C}^{*}$. Précisons que si $E$ est de rang $n$, i.e., $\operatorname{dim} E_{x}=n$,

$$
\operatorname{det}\left(I+\frac{i}{2 \pi} \Omega\right)=1+c_{1}(\nabla)+\cdots+c_{n}(\nabla)=c(\nabla)
$$

avec $c_{i}(\nabla), 2 i$-forme complex sur $M$.

Lemma 1. Si $\nabla$ est une connexion hermitienne, $c_{i}(\nabla)$ sont des formes réelles sur $M$.

En effet, comme $\Omega+{ }^{t} \bar{\Omega}=0$

$$
\operatorname{det}\left(I+\frac{i \Omega}{2 \pi}\right)=\operatorname{det}\left(I-\frac{i^{t} \bar{\Omega}}{2 \pi}\right) .
$$

Or évidemment,

$$
\operatorname{det}\left(I-\frac{i^{t} \bar{\Omega}}{2 \pi}\right)=\operatorname{det}\left(I-\frac{i \bar{\Omega}}{2 \pi}\right)=\overline{\operatorname{det}\left(I+\frac{i \Omega}{2 \pi}\right)} .
$$

Donc

$$
\overline{\operatorname{det}\left(I+\frac{i \Omega}{2 \pi}\right)}=\operatorname{det}\left(I+\frac{i \Omega}{2 \pi}\right) .
$$


2. Nous allons nous restreindre à un ouvert $U$ de trivialisation de $E$. Soit donc $\left(s^{1}, \cdots, s^{n}\right)$ une base locale de $E$ sur $U$. $K=\left(\Omega_{j}^{i}\right)$ étant la matrice représentative de $\Omega$, et $\left(\delta_{j}^{i}\right)$ celle de $I$,

$$
\begin{aligned}
P[\Omega] & =\operatorname{det}\left(I+\frac{i}{2 \pi} \Omega\right) \\
& =P\left[\delta_{1}^{1}+\frac{i}{2 \pi} \Omega_{1}^{i}, \cdots, \delta_{n}^{n}+\frac{i}{2 \pi} \Omega_{n}^{n}\right]
\end{aligned}
$$

où $P \in A^{\text {paiR }}\left[X_{1}^{1}, \cdots, X_{n}^{n}\right]$ l'algèbre de polynomes de $n \times n$ variables à coefficients dans l'algèbre $A^{\text {paiR }}$ des formes extérieures de degré pair sur $U$. Nous pouvons aussi considérer $P \in A\left[X_{1}^{1}, \cdots, X_{n}^{n}\right]$ où $A$ est l'algèbre de toutes les formes extérieures complexes sur $U$.

Faisons cette convention: pour tout $\theta=\left(\theta_{1}, \cdots, \theta_{n}\right) \in A^{n}, P_{j}^{i}\left[\theta_{i}\right]$ est la valeur dans $A$ du polynome $P$ quand

$$
X_{h}^{k}=\delta_{h}^{k}+\frac{i}{2 \pi} \Omega_{h}^{k} \text { si } k \neq j
$$

et

$$
X_{i}^{j}=\frac{i}{2 \pi} \theta_{i} .
$$

Alors, $P$ étant de fait à coefficients complexes, i.e., 0 -formes constantes, il est clair que $d P\left[\Omega_{1}^{1}, \cdots, \Omega_{n}^{h}\right]=P_{j}^{i}\left[d \Omega_{i}^{j}\right]$ (remarquez la sommation dûe à la convention d'Einstein).

D'autre part, $\omega=\left(\omega_{j}^{i}\right)$ étant la matrice représentative de la connexion $\nabla$ et $P$ étant de fait multilinéaire relativement aux variables $X_{1}^{1}, \cdots, X_{n}^{h}$, on a par l'identité de Bianchi (Lemma 2 de I et la remarque qui le suit).

$$
\begin{aligned}
P_{j}^{i}\left[d \Omega_{i}^{j}\right]+P_{j}^{i}\left[\omega_{k}^{j} \Lambda \Omega_{i}^{k}-\Omega_{k}^{j} \Lambda \omega_{i}^{k}\right] & =P_{j}^{i}\left[d \Omega_{i}^{j}+\omega_{k}^{j} \Lambda \Omega_{i}^{k}-\Omega_{k}^{j} \Lambda \omega_{i}^{k}\right] \\
& =\sum_{i, j=1}^{n} P_{j}^{i}[0]=0 .
\end{aligned}
$$

Or d'après une propriété élémentaire du det, on a

Donc

$$
P_{j}^{i}\left[\omega_{k}^{j} \Lambda \Omega_{i}^{k}-\Omega_{k}^{j} \Lambda \omega_{i}^{k}\right]=0 .
$$

$$
d P[\Omega]=P_{j}^{i}\left[d \Omega_{i}^{j}\right]=0
$$

de cette propriété locale, on a immédiatement

Proposition 1.

$$
d c(\nabla)=d\left[\operatorname{det}\left(I+\frac{i}{2 \pi} \Omega\right)\right]=0 .
$$

3. Soit donnée une famille de connexions $\nabla_{t}$ de $E$. Relativement 
à la base locale donnée $\left(s^{1}, \cdots, s^{n}\right)$ de $E,\left(\Omega_{j}^{i}(t)\right),\left(\omega_{j}^{i}(t)\right),\left(\dot{\omega}_{j}^{i}(t)\right)$ désigneront respectivement les matrices représentatives de $\Omega_{t}, \nabla_{t}$ et $\dot{\nabla}_{t}$. Avec la convention précédente de notation

$$
\begin{aligned}
P_{j}^{i}\left[\dot{\omega}_{i}^{j}(t)\right] & =Q\left[\Omega(t), \dot{\omega}_{1}^{1}(t)\right] \\
& =Q\left[\delta_{1}^{1}+\frac{i}{2 \pi} \Omega_{1}^{1}(t), \cdots, \delta_{n}^{n}+\frac{i}{2 \pi} \Omega_{n}^{n}(t), \frac{i}{2 \pi} \dot{\omega}_{1}^{1}(t), \cdots, \frac{i}{2 \pi} \dot{\omega}_{n}^{n}(t)\right]
\end{aligned}
$$

avec

$$
Q \in A\left[X_{1}^{1}, \cdots, X_{n}^{n}, X_{n+1}^{n+1}, \cdots, X_{n+n}^{n+n}\right],
$$

algèbre de polynomes de $2(n \times n)$ variables. Donc $\theta=\left(\theta_{1}, \cdots, \theta_{n}\right)$ prenons la même convention: $Q_{\beta}^{\alpha}\left[\theta_{\alpha}\right]$ désigne la valeur de $Q$ pour

$$
\begin{aligned}
& X_{j}^{i}=\delta_{j}^{i}+\frac{i}{2 \pi} \Omega_{j}^{i} \quad \text { si } \quad(i, j) \neq(\beta, \alpha) \\
& X_{n+j}^{n+i}=\frac{i}{2 \pi} \dot{\omega}_{j}^{i} \quad \text { si } \quad(n+i, n+j) \neq(\beta, \alpha) \\
& X_{\alpha}^{\beta}=\frac{i}{2 \pi} \theta_{\alpha} .
\end{aligned}
$$

Alors

( i ) $d Q[\Omega(t), \dot{\omega}(t)]=Q_{j}^{i}\left[d \Omega_{i}^{j}(t)\right]+Q_{n+j}^{n+i}\left[d \dot{\omega}_{i}^{j}(t)\right]$.

Or d'après le Lemma 3 de I

(ii ) $\frac{\partial}{\partial t} P[\Omega(t)]=P_{j}^{i}\left[d \dot{\omega}_{i}^{j}(t)+\omega_{k}^{j}(t) \Lambda \dot{\omega}_{i}^{k}(t)-\dot{\omega}_{k}^{j}(t) \Lambda \omega_{i}^{k}(t)\right]$

$$
\begin{aligned}
= & Q_{n+j}^{n+i}\left[d \dot{\omega}_{i}^{j}(t)\right]+Q_{n+j}^{n+i}\left[\omega_{k}^{j}(t) \Lambda \omega_{i}^{k}(t)\right. \\
& \left.-\dot{\omega}_{k}^{j}(t) \Lambda \omega_{i}^{k}(t)\right] .
\end{aligned}
$$

Et d'après l'identité de Bianchi, $Q$ étant multilinéaire par rapport aux variables, on a

$$
Q_{j}^{i}\left[d \Omega_{i}^{j}(t)+\omega_{k}^{j}(t) \Lambda \Omega_{i}^{k}(t)-\Omega_{k}^{j}(t) \Lambda \omega_{i}^{k}(t)\right]=0 .
$$

Donc on a de (i) et (ii)

(iii) $\frac{\partial}{\partial t} P[\Omega(t)]=d P_{j}^{i}\left[\dot{\omega}_{i}^{j}(t)\right]+Q_{j}^{i}\left[\omega_{k}^{j}(t) \Lambda \Omega_{i}^{k}(t)-\Omega_{k}^{j}(t) \Lambda \omega_{i}^{k}(t)\right]$

$$
+Q_{n+i}^{n+i}\left[\omega_{k}^{j}(t) \Lambda \dot{\omega}_{j}^{k}(t)-\dot{\omega}_{k}^{k}(t) \Lambda \omega_{i}^{k}(t)\right] .
$$

Remarquons que

$$
Q[\Omega(t), \dot{\omega}(t)]=\frac{\partial}{\partial x} P[\Omega(t)+x \dot{\omega}(t)]_{x=0} .
$$

Donc encore d'après la propriété élémentaire déjà citée du déterminant, on a 


$$
Q_{j}^{i}\left[\omega_{k}^{j}(t) \Lambda \Omega_{i}^{k}(t)-\Omega_{k}^{j}(t) \Lambda \omega_{i}^{k}(t)\right]+Q_{n+i}^{n+i}\left[\omega_{k}^{j}(t) \Lambda \dot{\omega}_{i}^{k}(t)-\dot{\omega}_{k}^{j}(t) \Lambda \omega_{i}^{k}(t)=0 .\right.
$$

D'où la formule importante suivante dûe à $\mathrm{A}$. Weil

$$
\frac{\partial}{\partial t} P\left[\Omega_{1}^{1}(t), \cdots, \Omega_{n}^{n}(t)\right]=d P_{j}^{i}\left[\dot{\omega}_{i}^{j}(t)\right] .
$$

Il est immédiat de vérifier que la forme $\left.P_{j}^{i}\left[\dot{\omega}_{i}^{j}\right)\right]$ est indépendante du choix de la base locale. Donc $P_{j}^{i}\left[\dot{\omega}_{i}^{j}(t)\right]$ est la restriction sur $U$ d'une forme extérieure globale notée $P\left[\Omega_{t}, \dot{\nabla}_{t}\right]$ de $M$.

Proposition 2. Etant une famille de connexions $\nabla_{t}$

$$
c\left(\nabla_{1}\right)-c\left(\nabla_{0}\right)=d \int_{0}^{1} P\left[\Omega_{t}, \dot{\nabla}_{t}\right] d t .
$$

Cette proposition n'est autre que l'intégration de la formula (iv) de A. Weil.

4. Classes de Chern. On vérifie immédiatement qu'étant données deux connexions $\nabla_{0}$ et $\nabla_{1}$,

$$
\nabla_{t}=t \nabla_{1}+(1-t) \nabla_{0}
$$

est une famille de connexions reliant $\nabla_{0}$ et $\nabla_{1}$. Done d'après la proposition (1) et (2), étant donnée une connexion $\nabla$ dans $E$, la classe de cohomologie de $c(\nabla)$ est indépendante du choix de $\nabla$. Nous pouvons noter cette classe par $c(E)$, nommée classe de Chern de $E$. Remarquons que cette classe est réelle: $c(E) \in H^{p \alpha i R}(M, R)=\bigoplus_{p} H^{2 p}(M, R)$ comme d'après le Lemma 1 , on peut toujours prendre une connexion hermitienne dans $E$ et $c(\nabla)$ est alors une forme réelle.

Si $f$ est une application d'une variété $M^{\prime} \longrightarrow M$ désignons par $f^{\prime} E$ le fibré vectoriel complexe sur $M^{\prime}$ induit par $f$ :

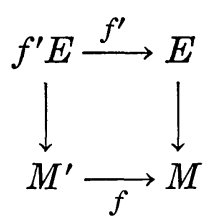

$f^{\prime}$ transporte de façon évidente toute section $s$ de $E$ en une section $f^{\prime} s$ de $f^{\prime} E$. Ceci dit, il est immédiat de vérifier que pour une connexion donnée $\nabla$ de $E$, il existe une et une seule connexion $\nabla^{\prime}$ sur $f^{\prime} E$ telle que

$$
\nabla^{\prime}\left(f^{\prime} s\right)=\left(f^{*} \otimes f^{\prime}\right)(\nabla(s))
$$

où $f^{*}$ est le prolongement de $f$ aux formes extérieures et $\left(f^{*} \otimes f^{\prime}\right)(\omega \otimes s)=\left(f^{*} \omega\right) \otimes f^{\prime} s$, comme section de $T_{C}^{*}\left(M^{\prime}\right) \otimes f^{\prime} E$. 
Nous avons alors

$$
c\left(\nabla^{\prime}\right)=f^{*} c(\nabla)
$$

ou encore par passage aux classes de cohomologie, on a la propriété universelle suivante de la classe de Chern

$$
c\left(f^{\prime} E\right)=f^{*} c(E) \text {. }
$$

\section{Propriétés géométriques des classes de Chern.}

1. Théorème de dualité de Whitney. Soit $E=E_{1} \oplus E_{2}$, somme directe de deux fibrés vectoriels complexes sur $M$. Si $\nabla_{1}$ et $\nabla_{2}$ sont des connexions respectivement dans $E_{1}$ et $E_{2}$,

$$
\begin{gathered}
\nabla=\nabla_{1} \oplus \nabla_{2}: E \longrightarrow T_{C}^{*} \otimes E=T_{c}^{*} \otimes E_{1} \oplus T_{C}^{*} \otimes E_{2} \\
s=s_{1}+s_{2} \longrightarrow \nabla(s)=\nabla_{1}\left(s_{1}\right)+\nabla_{2}\left(s_{2}\right)
\end{gathered}
$$

est évidemment une connexion dans $E$. Le tenseur de courbure $\Omega$ de $\nabla$ est tel que

$$
\begin{gathered}
\Omega=\nabla^{2}: E \longrightarrow \Lambda^{2} T_{C}^{*} \otimes E=\Lambda^{2} T_{C}^{*} \otimes E_{1} \oplus \Lambda^{2} T_{C}^{*} \otimes E_{2} \\
s=s_{1}+s_{2} \longrightarrow V^{2}(s)=\nabla_{1}^{2}\left(s_{1}\right)+\nabla_{2}^{2}\left(s_{2}\right) .
\end{gathered}
$$

Autrement dit,

$$
\Omega=\Omega_{1} \oplus \Omega_{2}
$$

Donc

$$
c(\nabla)=c\left(\nabla_{1}\right) \Lambda c\left(\nabla_{2}\right) .
$$

En passant aux classes de cohomologie, nous avons le théorème suivant dit de dualité de Whitney

THEOREM. $S i E=E_{1} \oplus E_{2}$, nous avons dans l'algèbre de cohomologie $H^{p \alpha i R}(M, R)$

$$
c(E)=c\left(E_{1}\right) c\left(E_{2}\right) \text {. }
$$

CoRollaire. Si $E$ admet une section partout non nulle on a $c_{n}(E)=0$ ( $n$ étant le rang de $\left.E\right)$. En effet, si $E$ admet une section partout non nulle $E=E_{1} \oplus C$, où $C$ est le fibré en droite trivial sur M. Donc

$$
c(E)=c\left(E_{1}\right)=1+c_{1}\left(E_{1}\right)+\cdots+c_{n-1}\left(E_{1}\right) .
$$

Remarque. Caractère de Chern. $A^{\text {paiR }}$ désigne l'algèbre extérieure de degré pair sur $M$. Donc l'algebre $A^{\text {paiR }}[X]$ des polynômes à coefficients dans $A^{p \alpha i R}$, posons pour une connexion $\nabla$ dans $E$

$$
c(\nabla)[X]=1+c_{1}(\nabla) X+\cdots+c_{n}(\nabla) X^{n} .
$$


Ecrivons formellement

$$
c(\nabla)[X]=\left(1+\gamma_{1} X\right) \cdots\left(1+\gamma_{n} X\right)
$$

alors

$$
\operatorname{ch}(\nabla)=e^{\gamma_{1}}+\cdots+e^{\gamma n}
$$

est un élement de $A^{\text {pair }}$. Des propriétés connues sur $c(\nabla)$, on conclut immédiatement que $\operatorname{ch}(\nabla)$ est une forme fermée, et que sa classe de cohomologie $c h(E)$, dite caractère de Chern de $E$, est définie indépendamment du choix de $\nabla$. Ceci dit,

(i) la théorème de dualité se traduit alors par la relation

$$
\operatorname{ch}\left(E_{1} \oplus E_{2}\right)=\operatorname{ch}\left(E_{1}\right)+\operatorname{ch}\left(E_{2}\right)
$$

(ii) étant donnés deux fibrés vectoriels complexes $E_{1}$ et $E_{2}$, munis respectivement des connexions $\nabla_{1}$ et $\nabla_{2}$ rappelons qu'on définit sur $E_{1} \oplus E_{2}$ une connexion canonique $\nabla$

$$
\begin{aligned}
\nabla: E_{1} \otimes E_{2} & \longrightarrow T_{C}^{*} \otimes E_{1} \otimes E_{2} \\
s_{1} \otimes s_{2} & \longrightarrow \nabla_{1}\left(s_{1}\right) \otimes s_{2}+s_{1} \otimes \nabla_{2}\left(s_{2}\right) .
\end{aligned}
$$

D'où

$$
\nabla^{2}\left(s_{1} \otimes s_{2}\right)=\nabla_{1}^{2}\left(s_{1}\right) \otimes s_{2}+s_{1} \otimes \nabla_{2}^{2}\left(s_{2}\right)
$$

ce qui entraine que si

$$
c\left(\nabla_{1}\right)[X]=\left(1+\gamma_{1} X\right) \cdots\left(1+\gamma_{p} X\right)
$$

et

$$
c\left(\nabla_{2}\right)[X]=\left(1+\lambda_{1} X\right) \cdots\left(1+\lambda_{q} X\right)
$$

on a

Done

$$
c(\nabla)[X)=\prod_{\substack{1 \leqq i \leqq p \\ 1 \leqq j \leqq q}}\left[1+\left(\gamma_{i}+\gamma_{j}\right) X\right]
$$

$$
\operatorname{ch}(\nabla)=\operatorname{ch}\left(\nabla_{1}\right) \operatorname{Ach}\left(\nabla_{2}\right)
$$

ou encore

$$
\operatorname{ch}\left(E_{1} \otimes E_{2}\right)=\operatorname{ch}\left(E_{1}\right) \cdot \operatorname{ch}\left(E_{2}\right) .
$$

Ainsi de façon un peu plus savante, $K$ étant le foncteur de Grothendieck de la catégorie des variétés différentiables à valeurs dans la catégorie des anneaux commutatifs, le caractère de Chern est une transformation naturelle de ce foncteur au foncteur qui associe à toute variété différentiable $M$ l'anneau commutatif $H^{p \alpha i k}(M, R)$ des classes de cohomologie de degré pair de $M$. 


\section{Théorème de Gauss-Bonnet.}

(i) L'indice de zéro d'une section. Considérons le fibré vectoriel complexe trivial $C^{n} \times C^{n}$ sur $C^{n}$, muni de l'orientation canonique. Une section $Z$ de ce fibré est une fonction différentiable

$$
Z: C^{n} \longrightarrow C^{n} \text {. }
$$

Supposons que $Z^{-1}(0)=0$; alors $Z$ applique $C^{n}-\{0\}$ dans lui-même. Or la sphère orientée $S^{2 n-1}=\left\{\left(z_{1}, \cdots, z_{n}\right) \in C^{n},\left|z_{1}\right|^{2}+\cdots+\left|z_{n}\right|^{2}=1\right\}$ est un retract par déformation de $C^{n}-\{0\} . Z$ est donc homotopiquement équivalent à une application que nous noterons encore par $Z$, de $S^{2 n-1}$ dans elle-même. D'où nous avons

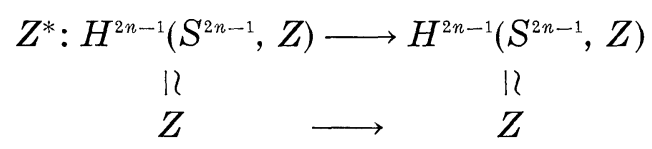

$Z^{*}$ est une multiplication par un entier algébrique Ind $(0, Z)$, appelé l'indice du zéro 0 de la section $Z$.

Exemple. Pour $n=1$,

$$
\text { Ind }(0, Z)=-\frac{i}{2 \pi} \int_{\gamma} \frac{d Z}{Z}
$$

où $\gamma$ est une courbe de Jourdan simple, rectifiable quelconque orientée dans le sens positif entourant l'origine 0 de $C$.

Dans le cas général où $E$ est un fibré vectoriel complexe de rang $n$ sur une variété $M$ orientée de dimension (réelle) $2 n$, pour une section $s$ de $E$ sur $M$ admettant $x$ comme un zéro isolé, on peut toujours se ramener au cas précédent sur un voisinage $U$ de trivialisation $E$, contenant $x$, difféomorphe à $C^{n}$ avec conservation de l'orientation et tel que $x$ corresponde à l'origine 0 de $C^{n}$. Et de cette manière, on définit l'indice Ind $(x, s)$ du zéro $x$ de la section $s$ de $E$.

(ii) Théorème de Gauss-Bonnet dans le cas du fibré de rang 1. Soit donc $E$ un fibré vectoriel complexe de rang 1 sur une surface compacte de Riemann, i.e., orientée et de dimension 2. Etant donnée une section $s$ quelconque à zéros isolés $x_{1}, \cdots, x_{p}$ de $E$ sur $M$, choisissons des voisinages de trivialization $U_{j}, 1 \leqq j \leqq p$, de $E$, contenant respectivement et seulement $x_{j}$. Par une partition de l'unité, on peut choisir une connexion $\nabla$ dans $E$, à courbure nulle sur les $U_{j}$. Désignons par $[M]$, la classe fondamentale d'homologie de dimension 2 de la variété orientée compacte $M$; nous avons

$$
\left\langle c_{1}(E),[M]\right\rangle=\int_{M} c_{1}(\nabla) .
$$


La connexion $\nabla$ étant à courbure nulle dans $U_{j}, c_{1}(\nabla)$ est une 2forme nulle à l'intérieur des disques $B\left(v_{j}\right)$ de centre $x_{j}$, contenus respectivement dans $U_{j}$ (i.e., des disques par un certain difféomorphisme conservant l'orientation de $U_{j}$ avec $C$ ); donc

$$
\int_{M} c_{1}(\nabla)=\int_{M \varepsilon} c_{1}(\nabla)
$$

avec $M_{\varepsilon}=M-\bigcup_{j} B\left(x_{j}\right)$.

Or sur $M_{\varepsilon}$, la section $s$ donnée est partout non nulle, peut être donc prise comme une base locale de $E$; on a sur $M_{\varepsilon}$

$$
\begin{aligned}
\nabla(s) & =\omega \otimes s, \omega \text { étant une 1-forme complexe sur } M_{\varepsilon} \cdot \\
\nabla^{2}(s) & =d \omega \otimes s-\omega \Lambda \nabla(s)=d \omega \otimes s-(\omega \Lambda \omega) \otimes s \\
& =d \omega \otimes s .
\end{aligned}
$$

Done sur $M_{\varepsilon}$,

$$
c_{1}(\nabla)=\operatorname{det}\left(\frac{i}{2 \pi} \Omega\right)=\frac{i}{2 \pi} d \omega .
$$

Par la formule de Stokes,

$$
\begin{aligned}
\int_{M \varepsilon} c_{1}(\nabla) & =\frac{i}{2 \pi} \int_{M \varepsilon} d \omega=\frac{i}{2 \pi} \int_{\partial M \varepsilon} \omega \\
& =-\sum_{j} \frac{i}{2 \pi} \int_{r j} \omega
\end{aligned}
$$

où $\gamma_{j}$ est la courbe de Jourdan simple orientée qui est le bord de $B\left(x_{j}\right)$.

D'autre part, rappelons que dans $U_{j}$, la connexion $\nabla$ est sans courbure. On peut supposer

$$
\nabla\left(e_{j}\right)=0 \text {, avec } e_{j} \text { une base locale de } E \text { sur } U_{j} \text {. }
$$

Dans $U_{j}$, la section $s$ étant de la forme

$$
s=z_{j} e_{j}, z_{j} \in \mathscr{C}^{\infty}\left(U_{j}, C\right)
$$

on a

$$
\nabla(s)=d z \otimes e_{j} .
$$

Ce qui fait que sur $\gamma_{j}$, bord de $B\left(x_{j}\right)$ contenu dans $U_{j}$,

$$
\nabla(S)=\omega \otimes s=\frac{d z_{j}}{z_{j}} \otimes s .
$$

Ainsi, 


$$
\left\langle c_{1}(E),[M]\right\rangle=-\sum_{j} \frac{i}{2 \pi} \int_{r_{j}} \frac{d z_{j}}{z_{j}}
$$

nous avons démontré le théorème dit de Gauss-Bonnet dans les hypothèses indiquées de $E$ et de $M$.

ThÉoRème DE Gauss-Bonnet. $\left\langle c_{1}(E),[M]\right\rangle=\sum_{j}$ Ind $\left(x_{j}, s\right)$, où les $x_{j}$ sont les zéros isolés d'une section quelconque $s$ du fibré $E$ de rang 1.

Le second membre de la dernière égalité étant un entier, le corollaire suivant est immédiat,

Corollaire. $c_{1}(E)$ est en fait une classe de cohomologie de $M$ à valeurs dans $Z$

$$
c_{1}(E) \in H^{2}(M, Z) \text {. }
$$

Exemple. Considérons l'espace projectif complexe $P_{n}(C)$, i.e., l'ensemble des sous espaces vectoriels $x$ de dimension 1 de $C^{n+1}$. Désignons par $H_{n}$, le sous fibré vectoriel complexe de rang 1 du fibré vectoriel trivial $P_{n}(C) \times C^{n+1}$ sur $P_{n}(C)$, formé des couples $(x, v)$ tels que $v$ soit un vecteur de $C^{n+1}$ contenu dans le sous espace $x$. $Q_{n}$ dénotera le fibré vectoriel quotient

$$
0 \longrightarrow H_{n} \longrightarrow P_{n}(C) \times C^{n+1} \longrightarrow Q_{n} \longrightarrow 0 \text {. }
$$

Considérons la section $s$ de $P_{1}(C) \times C^{2}$, telle que $s(x)=(x, v)$, où $v$ est un vecteur fixé non nul de $C^{2}$, la section $s$ définira au quotient une section $s^{\prime}$ de $Q_{1}$, qui adment un seul zéro $x=[v]$, le sous espace engendré par $v$. On peut voir facilement par un calcul direct que

$$
\text { Ind }\left([v], s^{\prime}\right)=1 \text {. }
$$

D'où par le théorème de Gauss-Bonnet,

$$
\left\langle c_{1}\left(Q_{1}\right),\left[P_{1}(C)\right]\right\rangle=1
$$

autrement dit, $c_{1}(Q)$ est la classe d'orientation canonique de $P_{1}(C)$. Par le théorème de dualité de Whitney,

$$
c_{1}\left(H_{1}\right)=-c_{1}\left(Q_{1}\right)
$$

$c_{1}(H)$ est la classe d'orientation opposée.

Rappelons que nous avons la décomposition cellulaire de $P_{n}(C)$ :

$$
P_{0}(C) \subset P_{1}(C) \subset \cdots \subset P_{n-1}(C) \subset P_{n}(C)
$$

par l'attachement à chaque étape une seule cellule de dimension $2 i$ 
correspondante et que $P_{n}(C)$ est une variété Kahlérienne. Donc,

$$
H^{*}\left(P_{n}(C), R\right)=R+R \omega+\cdots+R \omega^{n},
$$

l'algèbre de polynômes tronquée engendrée par la classe $\omega$ de la 2forme fondamentale Kahlérienne; et on pourrait démontrer aussi que

$$
H^{*}\left(P_{n}(C), Z\right)=Z+Z \zeta+\cdots+Z \zeta^{n},
$$

l'anneau de polynômes tronqué engendré par une classe $\zeta$ de degré 2 , telle que $\zeta^{n}$ soit la classe d'orientation canonique de $P_{n}(C)$. La classe $\zeta$ est aussi l'unique classe de degré 2 de $P_{n}(C)$, qui induit sur la sousvariété $P_{1}(C)$ de $P_{n}(C)$ sa classe d'orientation canonique; autrement dit, nous avons nécessairement

$$
c_{1}\left(H_{n}\right)=-\zeta \text {. }
$$

Encore par le théorème de dualité de Whitney,

$$
c_{n}\left(Q_{n}\right)=(-1)^{n} c_{1}\left(H_{n}\right)^{n}=\zeta^{n}
$$

autrement dit, pour tout entier $n, c_{n}(Q)$ est la classe d'orientation canonique de $P_{n}(C)$.

(iii) Théorème de Gauss-Bonnet dans le cas général. Nous considérons donc plus généralement un fibré vectoriel complexe $E$ de rang $n$ sur une variété compacte $M$ orientée et de dimension $2 n$. Pour simplifier, nous noterons par $E^{\prime}$ le fribé vectoriel induit $\pi^{\prime} E$ sur $E$ par la fibration $\pi: E \rightarrow M ; E^{\prime} n^{\prime}$ est autre que le produit direct de Whitney $E \times{ }_{M} E$, muni de la première projection sur $E$. Inversement si nous désignons par $j$ la section nulle de $E$ sur $M$, on a $E=j^{\prime} E^{\prime}$. Aussi, comme toute section $s$ de $E$ sur $M$ est homotope à la section $j, s^{\prime} E^{\prime}$ est isomorphe au fibré $E$ sur $M$. En particulier, pour toute connexion $\nabla^{\prime}$ dans $E^{\prime} s^{*} c_{n}\left(\nabla^{\prime}\right)$ définit la classe de cohomologie $c_{n}(E)$ de $M$ :

$$
\left\langle c_{n}(E),[M]\right\rangle=\int_{M} s^{*} c_{n}\left(\nabla^{\prime}\right)
$$

où $[M]$ est la classe d'homologie fondamentale de dimension $2 n$ de la variété compacte orientée $M$.

Nous supposerons donnée une section $s$ de $E$ sur $M$ à zéros isolés $x_{1}, \cdots, x_{p}{ }^{1}$ et choisissons comme précédemment des ouverts $U_{j}, 1 \leqq$ $j \leqq p$ de trivialisation de $E$, contenant respectivement et seulement $x_{j}$. $\quad U_{j}$ étant difféomorphe à $C^{n}$ avec conservation de l'orientation, nous désignerons par $B\left(x_{j}\right)$ le disque de centre $x_{j}$ dans $U_{j}$. Nous avons

1 Il existe toujours en effet dans le fibré vectoriel $E$ des sections à zéros isolés (voir N. Steenrod, Topology of fibre bundles), Princeton Univ. Press, 1951. 


$$
\int_{M} s^{*} c_{n}\left(\nabla^{\prime}\right)=\int_{M \varepsilon} s^{*} c_{n}\left(\nabla^{\prime}\right)+\sum_{j} \int_{B\left(x_{j}\right)} s^{*} c_{n}\left(\nabla^{\prime}\right)
$$

où $M_{\varepsilon}=M-\mathrm{U}_{j} B\left(x_{j}\right)$. Or il existe une section canonique $\sigma$ dans $E^{\prime}$ :

$$
\begin{gathered}
E^{\prime}=E_{M} \times E \\
\forall e \in E, \sigma(e)=(e, e) .
\end{gathered}
$$

La section $\sigma$ est partout non nulle sur $E_{0}=E-j(M), j$ étant la section null de $E$. Donc sur $E_{0}, E^{\prime}=H \oplus Q$, ou $H$ est le saus fibré trivial engendré par $\sigma$. En dehors d'un petit voisinage de $j(M)$, prenons $\Delta^{\prime}=\Delta_{H}+\Delta_{Q}$. Si $\Delta_{H}(\sigma)=\omega=\omega \otimes \sigma$, on a, loin de $j(M)$,

$$
\begin{aligned}
c_{n}\left(\Delta^{\prime}\right) & =\frac{i}{2 \pi} d \omega \wedge c_{n-1}\left(\Delta_{Q}\right) \\
& =\frac{i}{2 \pi} d\left[\omega \wedge c_{n-1}\left(\Delta_{Q}\right)\right]=d \alpha
\end{aligned}
$$

Par la formule de Stokes, nous avons donc

$$
\begin{aligned}
\int_{M} s^{*} c_{n}\left(\nabla^{\prime}\right) & =\int_{\partial \hat{M}} s^{*} \alpha+\sum_{j} \int_{B\left(x_{i}\right)} s^{*} c_{n}\left(\nabla^{\prime}\right) \\
& =\sum_{j}\left(-\int_{\gamma_{j}} s^{*} \alpha+\int_{B\left(x_{j}\right)} s^{*} c_{n}\left(\nabla^{\prime}\right)\right)
\end{aligned}
$$

où $\gamma_{j}$ est la sphère orientée, bord de $B\left(x_{j}\right)$. Cette formule montre qu'il nous suffit de faire une étude locale dans $U_{j}$, sur lequel $E$ n'est autre le fibré trivial $C^{n} \times U_{j}$ et $E^{\prime}$, le fibré $C^{n} \times C^{n} \times U_{j}$.

(a) Posons $C_{0}^{n}=C^{n}-\{0\}$. Sur $C_{0}^{n}$, le fibré trivial $C^{n} \times C_{0}^{n}$ est la somme directe de deux fibrés

$$
C^{n} \times C_{0}^{n}=[\sigma]+\pi^{\prime} Q_{n-1},
$$

où $\pi^{\prime} Q_{n-1}$ est le fibré induit de $Q_{n-1}$ par la projection canonique

$$
\pi: C_{0}^{n} \longrightarrow P_{n-1}(C)
$$

et $[\sigma]$ est le sous fibré de rang 1 de $C^{n} \times C_{0}^{n}$ engendré par la section

$$
\begin{aligned}
& \sigma: C_{0}^{n} \longrightarrow C^{n} \\
& \sigma\left(z_{1}, \cdots, z_{n}\right)=\left(z_{1}, \cdots, z_{n}\right) .
\end{aligned}
$$

Désignons par $\nabla_{Q}$ une connexion quelconque dans $Q_{n-1}$ et par $\nabla_{H}$ la connexion dans $[\sigma]$ telle que $\partial$ étant la dérivation complexe de Dolbeaut

$$
\nabla_{H}(\sigma)=\frac{\partial|z|^{2}}{|z|^{2}} \otimes \sigma
$$

avec $|z|^{2}=\left|z_{1}\right|^{2}+\cdots+\left|z_{n}\right|^{2}$. Prenons dans $C^{n} \times C_{0}^{n}$, la connexion

$$
\nabla_{0}=\nabla_{H}+\pi^{\prime} \nabla_{Q},
$$


où $\pi^{\prime} \nabla_{Q}$ est la connexion induite de $\nabla_{Q}$. On a

$$
c_{n}\left(\nabla_{0}\right)=\frac{i}{2 \pi} d\left(\frac{\partial|z|^{2}}{|z|^{2}} \Lambda \pi^{*} c_{n-1}\left(\nabla_{Q}\right)\right) .
$$

Vue que $c_{n-1}\left(\nabla_{Q}\right)$ est la classe d'orientation canonique de $P_{n-1}((C)$, il n'est pas difficile de montrer que

$$
-\frac{i}{2 \pi} \frac{\partial|z|^{2}}{|z|^{2}} \Lambda \pi^{*} c_{n-1}\left(\nabla_{Q}\right)
$$

est la classe d'orientation de $C_{0}^{n}$ (i.e., restreinte sur toute sphère de centre 0 dans $C^{n}$, elle définit la classe d'orientation canonique de cette sphère).

(b) Dans le fibré trivial $C^{n} \times C^{n}$ sur $C^{n}$, la connexion triviale sera notée par $\nabla_{1}$. Soit $\varphi$ une fonction positive sur $C^{n}$ telle que

$$
\begin{array}{lll}
\varphi(z)=1 & \text { si } & |z|>r \\
\varphi(z)=0 & \text { si } & |z|<1 / 2 r .
\end{array}
$$

De façon évidente, $\nabla_{t}=\varphi \nabla_{0}+(1-\varphi) \nabla_{1}$ définit une connexion dans le fibré $C^{n} \times C^{n}$. Désignons par $e^{1}, \cdots, e^{n}$ la base canonique du fibré trivial

$$
\nabla_{t}^{2}\left(e^{i}\right)=\left[d\left(\varphi \omega_{j}^{i}\right)-\varphi^{2} \omega_{k}^{i} \Lambda \omega_{j}^{k}\right] \otimes e^{j}
$$

$\left(\omega_{j}^{i}\right)$ étant la matrice représentative de le connexion $\nabla_{0}$, i.e.,

$$
\nabla_{0}\left(e^{i}\right)=\omega_{j}^{i} \otimes e^{j},
$$

$\omega_{j}^{i}$ des 1-formes complexes sur $C_{0}^{n}$. Ainsi

$$
c_{n}\left(\nabla_{t}\right)=\operatorname{det}\left(\frac{i}{2 \pi}\left[d\left(\varphi \omega_{j}^{i}\right)-\varphi^{2} \omega_{k}^{i} \Lambda \omega_{j}^{k}\right]\right) .
$$

(c) Rappelons que sur $U_{j}, E^{\prime}=C^{n} \times C^{n} \times U_{j}$. Nous pouvons supposer par une partition de l'unité que la connexion $\nabla^{\prime}$ choisie dans $E^{\prime}$, restreinte sur $U_{j}$, est la connexion naturelle induite par $\nabla_{t}$ dans $C^{n} \times C^{n}$ sur $C^{n} \times C^{n} \times U_{j}$. Alors la section $s$ donnée n'est autre qu'une application $s: U_{j} \longrightarrow C^{n}$. Supposons que la fonction $\varphi$ précédente soit choisie telle que

$$
s^{-1}(\{|z|<r\}) \subset B\left(x_{j}\right) .
$$

Nous avons

$$
\int_{r_{j}} s^{*} \alpha=-\frac{i}{2 \pi} \int_{\gamma_{j}} s^{*}\left(\frac{\partial|z|^{2}}{|z|^{2}} \Lambda \pi^{*} c_{n-1}\left(\nabla_{Q}\right)\right) .
$$

Donc, vue la remarque de la fin de la section (a) de notre démonstration 


$$
\int_{r_{j}} s^{*} \alpha=\operatorname{Ind}\left(x_{j}, s\right)
$$

D'autre part

$$
\int_{B\left(x_{j}\right)} s^{*} c_{n}\left(\nabla^{\prime}\right)=\int_{B\left(x_{j}\right)} s^{*} \operatorname{det}\left(\frac{i}{2 \pi}\left[d\left(\varphi \omega_{j}^{i}\right)-\varphi^{2} \omega_{k}^{i} \Lambda \omega_{j}^{k}\right]\right) .
$$

Tout en sachant qu'on doit choisir la fonction $\varphi$ telle que $1-\varphi$ soit à support dans $B\left(x_{j}\right)$, il est immédiat de montrer que

$$
\lim \int_{B\left(x_{j}\right)} s^{*} \operatorname{det}\left(\frac{i}{2 \pi}\left[d\left(\varphi \omega_{j}^{i}\right)-\varphi^{2} \omega_{k}^{i} \Lambda \omega_{j}^{k}\right]\right)=0
$$

quand $B\left(x_{j}\right)$ parcourt un filtre de voisinages de $x_{j}$. Autrement dit nous avons prouvé que

$$
\left\langle c_{n}(E),[M]\right\rangle=\sum_{j} \operatorname{Ind}\left(x_{j}, s\right)+\varepsilon
$$

avec $\varepsilon$ aussi petit qu'on veut. D'où le théorème

THÉORÈME GÉNÉRAL DE GAUSS-BonNET. E étant un fibré complexe de rang $n$ sur une variété compacte orientée $M$ de dimension $2 n$

$$
\left\langle c_{n}(E),[M]\right\rangle=\sum_{j} \text { Ind }\left(x_{j}, s\right)
$$

où les $x_{j}$ sont les zéros (isolés) d'une section $s$ de $E$ sur $M$.

CoRollaire. Les classes de Chern sont des classes rationnelles:

$$
\forall_{p}, c_{p}(E) \in\left(H^{2 p}(M, Q)\right) .
$$

En effet, d'après les résultats de $\mathrm{R}$. Thom, la cohomologie rationnelle de $M$ peut être définie à partir des cycles fermés qui soient des sous variétés de $M$. Soit donc donné un tel cycle $N$ de dimension $2 p$. Pour tout fibré $E$ sur $M, E \mid N$ est "stablement équivalent" à un fibré $E^{\prime}$ de rang $p$ sur $N$ :

$$
\left\langle c_{p}(E),[N]\right\rangle=\left\langle c_{p}\left(E^{\prime}\right),[N]\right\rangle .
$$

Or d'après le théorème de Gauss-Bonnet, le second membre est un entier.

Remarque. (1) Nous avons développé la théorie des classes earactéristiques de Chern dans la catégorie des variétés différentiables. Il est à rappeler que tout espace fibré sur un espace topologique paracompact de dimension cohomologique finie est l'induit par une 
application continue d'un fibré différentiable $E$ sur une variété Grassmanienne. Vue la propriéte universelle des classes de Chern, précisée dans II-4, il est alors immédiat d'étendre la théorie à la catégorie des espaces topologiques "admissibles" au sens de Hirzebruch (voir notre introduction).

(2) De fait le dernier corollaire n'est qu'un résultat partiel. On pourrait en effet démontrer à l'aide d'un argument de A. Borel (voir Hirzebruch, Topological methods in algebraic geometry) que les classes de Chern sont intégrales:

$$
c(E) \in H^{*}(M, Z) .
$$

Received November 11, 1969.

UNIVERSITÉ DE MONTRÉAL 


\section{PACIFIC JOURNAL OF MATHEMATICS}

\section{EDITORS}

H. SAMelson

Stanford University

Stanford, California 94305

\section{Richard PIERCE}

University of Washington

Seattle, Washington 98105
J. DugundjI

Department of Mathematics

University of Southern California

Los Angeles, California 90007

BASIL GoRDON*

University of California

Los Angeles, California 90024

\section{ASSOCIATE EDITORS}
E. F. BECKENBACH
B. H. NeumanN
F. WOLE
K. YosHIDA

\section{SUPPORTING INSTITUTIONS}

UNIVERSITY OF BRITISH COLUMBIA

CALIFORNIA INSTITUTE OF TECHNOLOGY

UNIVERSITY OF CALIFORNIA

MONTANA STATE UNIVERSITY

UNIVERSITY OF NEVADA

NEW MEXICO STATE UNIVERSITY

OREGON STATE UNIVERSITY

UNIVERSITY OF OREGON

OSAKA UNIVERSITY

UNIVERSITY OF SOUTHERN CALIFORNIA

\author{
STANFORD UNIVERSITY \\ UNIVERSITY OF TOKYO \\ UNIVERSITY OF UTAH \\ WASHINGTON STATE UNIVERSITY \\ UNIVERSITY OF WASHINGTON \\ * * * * \\ AMERICAN MATHEMATICAL SOCIETY \\ CHEVRON RESEARCH CORPORATION \\ TRW SYSTEMS \\ NAVAL WEAPONS CENTER
}

The Supporting Institutions listed above contribute to the cost of publication of this Journal, but they are not owners or publishers and have no responsibility for its content or policies.

Mathematical papers intended for publication in the Pacific Journal of Mathematics should be in typed form or offset-reproduced, (not dittoed), double spaced with large margins. Underline Greek letters in red, German in green, and script in blue. The first paragraph or two must be capable of being used separately as a synopsis of the entire paper. The editorial "we" must not be used in the synopsis, and items of the bibliography should not be cited there unless absolutely necessary, in which case they must be identified by author and Journal, rather than by item number. Manuscripts, in duplicate if possible, may be sent to any one of the four editors. Please classify according to the scheme of Math. Rev. 36, 1539-1546. All other communications to the editors should be addressed to the managing editor, Richard Arens, University of California, Los Angeles, California, 90024.

50 reprints are provided free for each article; additional copies may be obtained at cost in multiples of 50 .

The Pacific Journal of Mathematics is published monthly. Effective with Volume 16 the price per volume (3 numbers) is $\$ 8.00$; single issues, $\$ 3.00$. Special price for current issues to individual faculty members of supporting institutions and to individual members of the American Mathematical Society: $\$ 4.00$ per volume; single issues $\$ 1.50$. Back numbers are available.

Subscriptions, orders for back numbers, and changes of address should be sent to Pacific Journal of Mathematics, 103 Highland Boulevard, Berkeley, California, 94708.

PUBLISHED BY PACIFIC JOURNAL OF MATHEMATICS, A NON-PROFIT CORPORATION

Printed at Kokusai Bunken Insatsusha (International Academic Printing Co., Ltd.), 7-17, Fujimi 2-chome, Chiyoda-ku, Tokyo, Japan.

* Acting Managing Editor. 


\section{Pacific Journal of Mathematics}

\section{Vol. 33, No. $2 \quad$ April, 1970}

Raymond Balbes and Alfred Horn, Projective distributive lattices ....... 273

John Findley Berglund, On extending almost periodic functions ......... 281

Günter Krause, Admissible modules and a characterization of reduced left

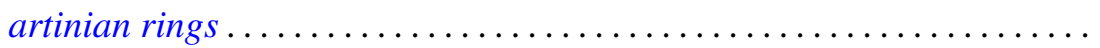

Edward Milton Landesman and Alan Cecil Lazer, Linear eigenvalues and a nonlinear boundary value problem ....................... 311

Anthony To-Ming Lau, Extremely amenable algebras ............... 329

Aldo Joram Lazar, Sections and subsets of simplexes .............. 337

Vincent Mancuso, Mesocompactness and related properties ............. 345

Edwin Leroy Marsden, Jr., The commutator and solvability in a generalized orthomodular lattice .................................. 357

Shozo Matsuura, Bergman kernel functions and the three types of canonical domains.......................................... 363

S. Mukhoti, Theorems on Cesàro summability of series .............. 385

Ngô Van Quê, Classes de Chern et théorème de Gauss-Bonnet ........... 393

Ralph Tyrrell Rockafellar, Generalized Hamiltonian equations for convex problems of Lagrange ................................ 411

Ken iti Sato, On dispersive operators in Banach lattices ............. 429

Charles Andrew Swanson, Comparison theorems for elliptic differential

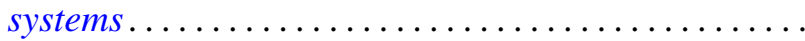

John Griggs Thompson, Nonsolvable finite groups all of whose local subgroups are solvable. II

David J. Winter, Cartan subalgebras of a Lie algebra and its ideals ... 